\title{
Tracking intruder ship in wireless environment
}

\author{
Madhuri Rao ${ }^{1 *}$ (ib and Narendra Kumar Kamila²
}

\author{
${ }^{*}$ Correspondence: \\ madhurirao@soauniversity.ac.in \\ ${ }^{1}$ Department of Computer \\ Science \& Engineering, \\ Institute of Technical \\ Education \& Research, Siksha \\ 'O'Anusandhan University, \\ Bhubaneswar, Odisha, India \\ Full list of author information \\ is available at the end of the \\ article
}

\begin{abstract}
It is a challenging task for all Harbors or Naval Administration to restrict and monitor the movement of defense or commercial ships. Most commonly used techniques of monitoring are radars and satellite images. These techniques are not reliable as radars can be turned off voluntarily and receptions of images are affected by adverse climatic conditions. This paper proposes a reliable ship intruder detection algorithm that classifies different types of objects approaching the model system in and out of phase with the ocean waves. The proposed technique also takes care of superimposition of temporal and spatial values of nodes that are presumably deployed in the sea surface up to a certain distance. Simulation results prove that the proposed algorithm detects and classifies objects efficiently even when $50 \%$ of the nodes reporting the tracking phenomenon are tampered.
\end{abstract}

Keywords: Intruder detection, Superimposition, Temporal and spatial values, Tracking objects, Wireless sensor network (WSN)

\section{Background}

Object tracking is one of the most practical applications of WSN. Wireless network is itself vulnerable and when combined with sensors the other errors that add upon to it are sensor failures, localization errors, prediction, and detection errors. Robust and effective solutions that could recover the information of tracking object are required for making wireless sensor networks more acceptable. Efficient energy management and enhanced network lifetime are other desired design aspects of a wireless sensor network application. Rout and Ghosh [1] tackle the problem of the area around the sink node that is a bottleneck zone as more activity is reported there, causing improper load balance that affects the overall network lifetime. They attempted to solve this problem by proposing a duty cycled wireless sensor network. The sensor nodes in the bottleneck zone are divided into two groups in their approach: simple relay sensors and network coder sensors. While the relay nodes simply forward the received data, the network coder nodes transmit using a network coding based algorithm. Energy efficiency of the bottleneck zone hence increases as more volume of data will be transmitted to the Sink with the same number of transmissions. Park and Shi [2] suggest strategies of deploying additional sensors in the bottleneck zone in due course of time to maintain a desired level of network connectivity. The major concerns of WSNs are studied by Mohsin [3] who also

(c) The Author(s) 2017. This article is distributed under the terms of the Creative Commons Attribution 4.0 International License (http://creativecommons.org/licenses/by/4.0/), which permits unrestricted use, distribution, and reproduction in any medium, provided you give appropriate credit to the original author(s) and the source, provide a link to the Creative Commons license, and indicate if changes were made. 
survey the performance tradeoffs in terms of energy, tracking error and other performance metrics of the available sensors in established network architectures. Tracking and monitoring techniques need to be designed by taking account of these parameters. A simple and efficient tracking system based on Oriented FAST and Rotated BRIEF (ORB) is presented by Wu et al. [4]. It is a fast binary descriptor where good matched points are obtained by computing Hamming distance. Based on the matched points and temporal-spatial constraint, location of the object in new frame is obtained. Tracking applications of wireless sensor network attract the need for a lot of research.

Tracking an intruder enemy vehicle in a battlefield and tracking an intruder ship on the surface of a sea differ in every aspect. The paper explores early ship intruder detection systems here. Ships can be equipped with wireless sensor networks. Kdouh et al. [5] propose a wireless sensor network on a boat for tracking objects continuously. The network collectively reports the position of various objects in the ship, in terms of Cartesian coordinates to a sink node or to a central base station. This application of wireless sensor network reduces the amount of cables and communication equipment on a ship and makes it less messy than a system with wired connections of embedded sensors. However detection of intruding objects was not a part of their research. Thattil and Vasantha [6] proposed an early energy efficient tracking technique that detects an intruder approaching a military border, with the help of wireless sensor network. However their technique cannot be applied for detecting intruders like a pirate boat, smugglers and illegal fisherman, freely moving on ocean and open waters. Monitoring and Tracking on a sea surface needs a different insight in itself. Luo et al. [7] have proposed a solution for ship intrusion detection, where three-axis accelerometer are deployed in an experimental WSN on the sea's surface to distinguish between authorized and unauthorized ships, it is not sufficient as they do not explore the possibilities of how energy consumption could be reduced. Their proposed system is not at all fault tolerant.

$\mathrm{Li}$ and Parker [8] proposed an unsupervised fuzzy adaptive resonance theory neural network to learn and detect intruders in a previously unknown environment that is equipped with wireless sensor network for tracking and monitoring. The detection of network intrusion itself is a very important task of the network security in wireless sensor network applications. Every task in wireless environment requires energy that is limited. Effective and optimal intruder detection techniques are required for wireless sensor network applications which are impacted by the area of their deployment. Intruder detection techniques that work optimal in a particular application may be absolutely inefficient for another application under wireless sensor network environment in sea.

\section{Related work}

Terrestrial monitoring using wireless sensor network technique is being carried out as a part of military surveillance. But monitoring the sea is always more challenging task for sensor networking scientists. In present scenarios terror attacks through harbor and open seas are increasing rapidly. Managing such an environment requires advanced technology that could alert the base stations when invaded or trespassed. Reported news of illegal fisherman, pirates and smugglers and of unauthorized marine vessels in harbors and ocean across the globe is increasing. Compared with traditional ship detection methods which can monitor a large area with technologies such as radars and satellites 
cost a lot, and fail in harsh weathers and also these devices could be turned off if desired. Hence reliable techniques for detection of intruders on open waters are indeed requirement of the time. Luo et al. [7], proposed a ship intruder detection technique where a ship carries an array of sensors with itself. It uses spatial and temporal correlations of the amplitude data collected by these senor nodes to detect an approaching object by the help of the wave pattern generated by it. The design suggested by Luo. et al., cannot however, support online intrusion detection. Their approach can help classify objects moving in the vicinity, but cannot detect multiple objects of similar form, such as two or more pirate ships, etc.

The feasibility of wireless sensor networks on ships was studied by Kdouh et al. [5]. The metallic structure of ships/boats makes wireless communication more difficult than in any other classical indoor and outdoor environments. In this regard two types of experiments have been carried out on board of a ferry-type boat during sailings and stopovers. The first experiment consists of point-to-point measurements using Zigbee-based equipments and the second one consists of deploying and testing a WSN on the ferry. This particular environment is characterized by its metallic structure which may severely disturb the wireless communication. Here they have studied by conducting point-topoint measurements for the different configurations of communication between nodes in a ship (nodes in the same room, nodes in adjacent rooms or adjacent decks). It is one of the first steps towards replacing the wired shipboard monitoring system by the cost effective WSN technology. The network employs the object tracking techniques to continuously report the position of the object in terms of Cartesian coordinates to a sink node or to a central base station. A wide range of technologies, network architectures and types of sensors are available for tracking the object in wireless sensor network environment. Little attention has been given in tracking pirate/smuggler's approaching a ship on sea, which we explore in this paper. There has been significant research in maritime surveillance using synthetic aperture radar (SAR). Graziano et al. [9] have indeed developed an algorithm for estimating the direction a ship is heading and could also estimate the ship velocity using SAR images. However their approach is not helpful to detect ship intrusion from within a ship. Zhai et al. [10] propose a ship detection method based on anomaly detection theory of SAR images. However their method cannot be applied to wireless sensor network.

Table 1 summarizes the number of pirate attacks that have occurred region wise and the kind of ship that was attacked. Hastings [11] brings this information to the limelight. He has suggested that more stringent pirate punishment laws and advanced tracking technology are required to control such incidents. It definitely raises many serious questions about the current state of art of defense and surveillance systems provided by the ship manufacturers. Even after seas monitoring frequency have been enhanced, still the occurrences of mishaps have not been combated, rather magnified only Zhai et al. [10]. Tracking of the wave patterns generated by ships could help in tracking pirates and militants, for reporting and raising alarms. An initial model of how harbor surveillance can be made more effective with WSN was proposed by Rao et al. [12]. Existing monitoring and tracking system can be enhanced in much better way, as currently satellite and radar is the most used and available commercial method of monitoring and tracking on sea. Images from satellite and via radar cannot identify the following events on sea: 
Table 1 Pirate ship attack type and region

\begin{tabular}{lccccr}
\hline Region & Robberies & $\begin{array}{l}\text { Ship/cargo } \\
\text { seizures }\end{array}$ & $\begin{array}{l}\text { Kidnappings } \\
\text { for ransom }\end{array}$ & Attacks & Total \\
\hline Arabian Sea & 31 & 5 & 8 & 1 & 44 \\
East Africa & 119 & 3 & 191 & 5 & 319 \\
Indian Ocean & 510 & 13 & 29 & 5 & 319 \\
Malacca Strait & 145 & 20 & 1 & 3 & 197 \\
Mediterranean Sea & 22 & 4 & 1 & 0 & 27 \\
Other & 19 & 2 & 3 & 0 & 22 \\
South America & 473 & 4 & 31 & 1 & 481 \\
South China Sea & 1130 & 91 & 305 & 16 & 1257 \\
West Africa & 461 & 15 & & 46 & 516 \\
& 2911 & 157 & & & 3420 \\
\hline
\end{tabular}

1. Ships are identified as stationary if moving with a speed of less than 0.5 knots and otherwise as moving.

2. Ships as loaded or discharged.

3. Change of destination of ships or vessels.

4. Report when vessels are out of range.

Satellite and radar fail to monitor the area around a moving ship in sea. Many instances of ship hijacking have been reported. Ships are vulnerable when at sea to attacks by pirates and smugglers. Technology in addition to satellites and radars is needed in order to equip and alert the people on board of a ship when an event of trespassing is likely to happen. This is possible by monitoring the water surface area surrounding the ship. This is achieved with the help of wireless sensor network. The wireless sensor network needs the intelligence of classifying objects by identifying the pattern of waves generated by them. However, more research in this direction is required to overcome such problem and with the help of Wireless Sensor Network this could be achieved. None of the above authors have discussed about the problems which we are considering here.

\section{Motivation}

The number of instances of crimes, smuggling and terrorism is increasing day by day in open waters. Our ships on oceans and seas need self tracking and monitoring systems in addition to support received from radars and satellites. Wireless sensor network provides a viable solution either in the form grid like structure or mesh topology. A grid of sensors can move along with the ship to monitor the ocean area surrounding the moving ship. If an object like a small unidentified ship is traced in its vicinity, necessary alarms and self defensive measures can be taken by people on the ship. Tracking and classifying objects is possible with the help of understanding the size, shape and behavior of the waves deformed by objects. These motivated to tackle the challenges of identifying approaching objects on ocean surface by their waveforms with the help of wireless sensor network. Knowing the constraints of wireless sensor network, these challenges become complex to solve with the need of reducing energy consumption of nodes, reducing congestion, delay and jitter while also ensuring that object detection is accurate. 


\section{Contributions}

The contributions of our research can be categorized into two sets. The first set relates to the use of spatial and temporal correlation techniques for identifying various objects approaching a grid of sensors with the sink node situated at the centre of the grid. The waveforms generated when two ships approach and move away from each other are also taken into consideration for better results. These waveforms are sensed and reported to the sink node, which can raise an alarm for the Captain on the ship, if any other ship approaches it. Under this situation a waveform classification algorithm has been developed. To ensure that this waveform classification algorithm yields accurate results even when sensor nodes are tampered, we have developed a reliable and fault tolerant system that uses bitwise AND and OR operation on sensor nodes data. We demonstrate the percentage of success of accurate object detection with random number and pattern of tampered nodes. The object detection degrades when more than $50 \%$ of the nodes are tampered, which is quite natural and practical.

Secondly, we address the issue of congestion in the bottleneck zone which comprises of nodes immediately surrounding the sink node. These nodes traditionally keep forwarding packets to the sink node, without having a chance to go to sleep state. We propose a scheduled duty cycle for the nodes in the bottleneck zone in such a manner that fair load distribution is achieved without compromising traffic flow. Finally from the results it is observed that our technique reduces average jitter and enhances the throughput of the network.

\section{Problem formulation}

Consider a sensor topology of $n^{*} n$ nodes with the sink node deployed in the centre of this topology. The nodes are equipped with sensors that measure the amplitude of sea waves and report the changing amplitude values to the sink node. Different objects for example fish, snake, ship etc., generate different types of wave patterns causing sensor to report different values based on their behavior and size. Hence nodes report different patterns of raising amplitude values to the sink node. The sink node can thus perform a spatial and temporal correlation for searching a particular pattern of size and activity in the values reported to it. The sink node applies this technique to detect an intruding object such as a pirate ship. On detecting a pirate ship, the sink node can raise necessary alarms. It, therefore, requires learning and remembering a vast type of wave patterns including patterns caused when two ships approach and move away from each other. The topology under study is believed to be in dynamic equilibrium and is assumed to be moving along with the ship as a tool to protect itself from intruding ships and smugglers. This topology has two inherent constraints. Firstly, the nodes immediately surrounding the sink nodes are constantly forwarding packets to sink node, thereby draining their power quickly. This is the bottleneck zone, and here over performing nodes will die and leave the network disconnected. Secondly the sink node classifies objects around itself based on spatial and temporal correlation of sensor nodes value. But if the values reported by the nodes are tampered, or if the nodes are physically tampered; the sink node will fail to function. Yu et al. [13] have proposed a distributed fault detection algorithm for WSN. They have shown how faulty data causes inaccurate estimation of the environment and causing wastage of network resources. A reliable object classification and intruder detection technique is needed to be performed by the sink node so that 
object detection would be accurate. In this paper, these issues are addressed. The proposed algorithm has two phases: phase 1 aims to classify objects in a fault tolerant manner; phase 2 deals with the issue of load imbalance in the bottleneck zone.

In general, this problem can be expressed mathematically with the help of following notations (Table 2).

Object classification consists of associating each object, to the waveform it generates while moving on sea surface. The features of the waveform are based on the size, shape and behavior of the object. Let $\mathrm{X}$ be the $\mathrm{n}$-dimensional random variable corresponding the waveform features. A vector $x=\left(x_{1}, \ldots x_{n}\right)$ as indicated in Table 2 consisting of the $n$ measured features is associated to each object. Let us assume that there are $c$ numbers of objects to be identified. We further define a random variable $\mathrm{Y}$ that indicates the object that generates a particular waveform. It takes values in the set $\{1,2, \ldots, c+1\}$. $\mathrm{Y}=c+1$ means that the waveform is not associated to any object, i.e., it is unknown. The problem of object classification is to associate a given waveform $x$ with an object $y$. We further consider that a set of $\mathrm{F}$ nodes report faulty or erroneous data to sink node. This may result in modified vector $x^{\prime}$. Based on the spatial and temporal values of vector $x$ over time the object is still detected successfully. An intrusion is understood by the difference of vector $x^{\prime}$ and expected $x$. The sink node uses the proposed algorithm to classify objects and data to sink node is forwarded via a set of $\mathrm{R}$ active relay nodes, while $\mathrm{R}^{\prime}$ set of relay nodes are asleep to bring fair load distribution.

\section{Phase 1 of the algorithm: tracking waveform patterns in a fault tolerant manner}

Figure 1 shows the pattern of wave generated by two ships moving in parallel with same speed and velocity. Ursell [14] proved that the pattern of waves generated by ships is a $\mathrm{V}$-shaped pattern and is independent of the size and velocity of the ship. This V-shaped pattern is formed by two loci of cusps whose angle with the sailing line is $19^{\circ} 28^{\prime}$ in deep water and $54^{\circ} 44^{\prime}$ is the angle between the sailing line and the diverging wave crest line at the cusp of the local line. Figure 2a, b depicts the resultant waves that are created when two waves meet in and out of phase. Figure $2 \mathrm{c}$ illustrates the pattern of waves that are generated when two boats with same velocity approach each other from opposite direction.

Here, superposition principle is applied. If two or more waves pass through the same medium simultaneously, then the resultant wave's displacement at any point $\mathrm{x}$ can be known by adding displacements of the component waves.

Table 2 Notations used

\begin{tabular}{ll}
\hline Notations & Descriptions \\
\hline $\mathrm{N}, \mathrm{E}$ & Represents a set of nodes and a set of edges of the graph \\
$\mathrm{S}$ & Sink node deployed at the centre of a grid \\
$\mathrm{F}$ & A set of unknown faulty nodes or tampered nodes \\
$\mathrm{R}$ & A set of ACTIVE relay nodes in the bottleneck zone (area around the sink nodes) \\
$\mathrm{R}^{\prime}$ & Set of SLEEP relay nodes \\
Vector $\mathrm{x}$ & Consisting of $n$ measured features of size and shape of waveform created by objects \\
Vector $y$ & A set of objects \\
Vector $x^{\prime}$ & Modified set of vector $x$ due to faulty sensors \\
\hline
\end{tabular}




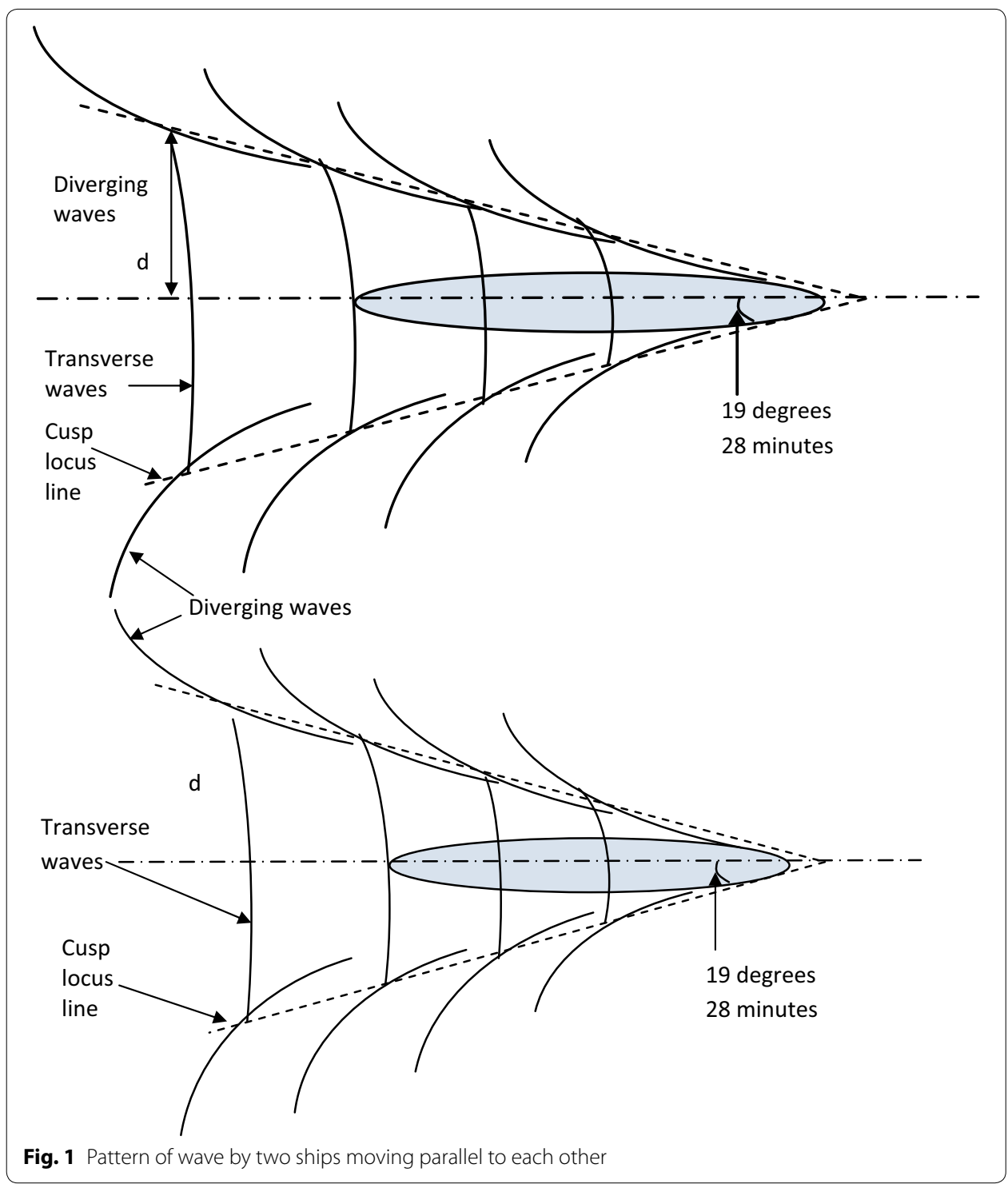

$$
\xi(x, t)=\xi_{1}(x, t)+\xi_{2}(x, t)
$$

$\xi_{1}$ is the displacement of first wave and $\xi_{2}$ is the displacement of the second wave where $\mathrm{x}$ is the amplitude and $\mathrm{t}$ is the given time.

When two ships are travelling with the same speed in same direction and if the amplitude and frequency are same as well, then the combined displacement of the wave is mathematically derived as follows:

$$
\xi(\mathrm{x}, \mathrm{t})=\mathrm{A}\left[\sin (\omega t-\mathrm{kx})+\sin \left(\omega t-\mathrm{kx}+\phi_{0}\right)\right]
$$

We take,

$$
\begin{aligned}
& \xi_{1}(\mathrm{x}, \mathrm{t})=\mathrm{A} \sin (\omega t-\mathrm{kx}) \\
& \xi_{2}(\mathrm{x}, \mathrm{t})=\mathrm{A} \sin \left(\omega t-\mathrm{kx}+\phi_{0}\right)
\end{aligned}
$$




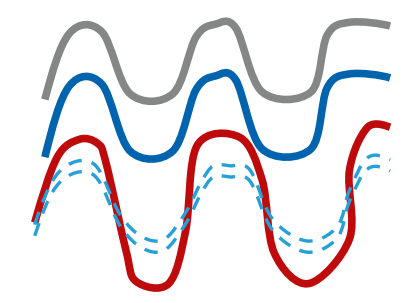

a Constructive interference of boat generated waves

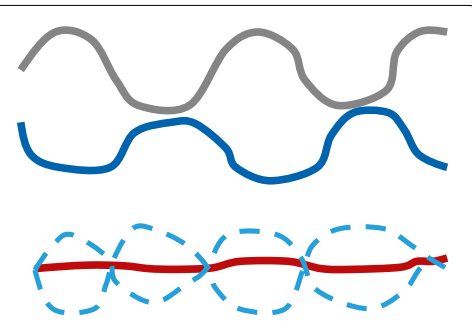

b Destructive interference of boat generated waves

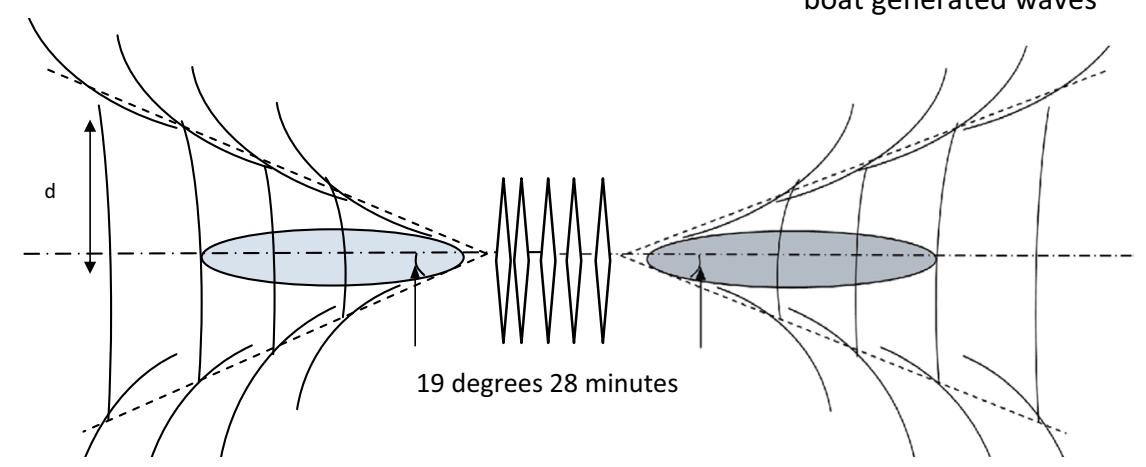

C Wave pattern generated when two boats with same velocity approach each other

Fig. 2 Waves generated when ships approach each other with same velocity

where $\omega=(2 \pi / T)$ is the angular frequency $\left(\right.$ in $\left.\operatorname{rad}^{-1}\right), k=(2 \pi / \lambda)$ is called the wave number (in $\mathrm{m}^{-1}$ ) and $\phi_{0}$ is phase constant. Moreover the argument of the sine function $(\omega t \pm k x)$ is called the phase of the wave. $T=$ time period, $\lambda=(1 / T)$ wavelength and $\mathrm{A}=$ amplitude of the wave which is the maximum displacement of any particle from its equilibrium position.

Since $\sin \alpha+\sin \beta=2 \sin (\alpha+\beta / 2) \cos (\alpha-\beta / 2)$

Then $\xi(\mathrm{x}, \mathrm{t})=2 \mathrm{~A} \sin \left(\omega t-\mathrm{kx}+\phi_{0} / 2\right) \cos \left(\phi_{0} / 2\right)$

If $\phi_{0}=0$ then $\xi(\mathrm{x}, \mathrm{t})=2 \mathrm{~A} \sin (\omega t-\mathrm{kx})$

If $\phi_{0}=\pi\left(\right.$ or $\left.180^{\circ}\right)$ then $\xi(x, t)=0$

If $\xi>A$, then it is called constructive interference.

And if $\xi<\mathrm{A}$, it is called destructive interference.

For two waves travelling in the opposite direction, with the same amplitude, frequency and speed, a standing wave is produced.

For the wave generated by the boat travelling to the right

$$
\xi_{\mathrm{R}}=\mathrm{A} \sin (\omega t-\mathrm{kx})
$$

For the wave generated by the boat travelling to the left

$$
\xi_{\mathrm{L}}=\mathrm{A} \sin (\omega t+\mathrm{kx})
$$


The resultant standing wave generated is

$$
\begin{aligned}
\xi & =\xi_{\mathrm{R}}+\xi_{\mathrm{L}}=\mathrm{A} \sin (\omega t-\mathrm{kx})+\mathrm{A} \sin (\omega t+\mathrm{kx}) \\
& =2 \mathrm{~A} \sin (\mathrm{kx}) \cos (\omega t)
\end{aligned}
$$

Any particle will vibrate with simple harmonic motion (SHM) due to the cos factor $(\omega t)$ and all will have the same frequency $f=\omega / 2 \pi$, but the amplitude depends on $x$ and equals $2 \mathrm{~A} \sin (\mathrm{kx})$.

The maximum amplitude or antinode occurs when $\xi=2 \mathrm{~A}$, that is when $\sin (\mathrm{kx})=1$ that is when $\mathrm{x}=\pi / 2 \mathrm{k}, 3 \pi / 2 \mathrm{k}, 5 \pi / 2 \mathrm{k}, \ldots$ or $\mathrm{x}=\lambda / 4,3 \lambda / 4,5 \lambda / 4, \ldots$

In general antinodes for displacement (or nodes for pressure) are observed when

$$
\mathrm{x}=(2 \mathrm{n}+1) \lambda / 4 \text { where } \mathrm{n}=0,1,2,3, \ldots
$$

Zero displacement or node occurs when

$$
\xi=0 \text {, that is when } \sin (\mathrm{kx})=0 \text {. }
$$

So nodes occur when $\mathrm{kx}=0, \pi, 2 \pi, 3 \pi, \ldots$ or when $\mathrm{x}=0, \lambda / 2, \lambda, 3 \lambda / 2$, etc.

In general nodes for displacement (or antinodes for pressure) are formed when

$$
\mathrm{x}=\mathrm{n} \lambda / 2 \text { where } \mathrm{n}=0,1,2,3 \text {, }
$$

The distance between nodes and antinodes is half a wavelength, $\lambda / 2$.

Chwang and Chen [15], show that at a fixed spatial point, the ship generated wave pattern has a limited duration. This temporal aspect of a ship generated wave helps in target detection. With reading reported by the nodes sensing the effect in which the transverse and diverging waves correlate, help the sink node to classify another ship approaching or leaving the surrounding. It could further help in identifying various objects approaching or leaving the surrounding, such as snake, fish or even a human being swimming to or away from the sink. Figure $2 \mathrm{c}$ depicts the pattern of waves that are generated when two boats with same velocity approach each other from opposite direction. This resultant standing wave is obtained as justified mathematically in Eq. 8. This correlation helps in tracking an unknown object or even a pirate ship. Spatial and temporal correlation has been explored in other areas as well. For instance, Ogawa et al. [16] have developed a system for differentiating knot-tying procedures in surgical procedures using a RGB-D Sensor. The sensor detects the various phases of the knot tying procedure by correlating the transition of hands spatially and temporally. He et al. [17] have studied the temporal and spatial characteristics of traffic variations in order to determine optimal deployment of base stations in green cellular networks which can thus reduce energy consumption. We would therefore, like to emphasize that objects could be classified based on their size, shape and behavior. The classification algorithm uses the concept of decision trees for training and the pseudo code is as follows: 


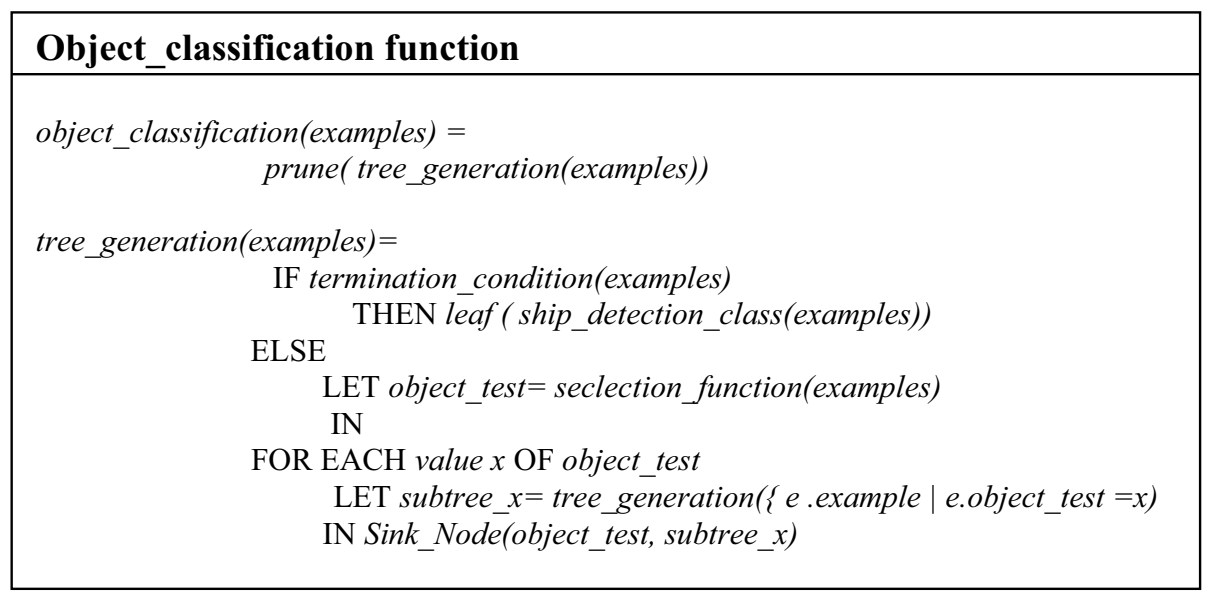

The algorithm runs recursively on each child node of a decision tree where a branch is selected based on the result of the logical test of the attributes (spatial and temporal). Vector $x=\left(x_{1}, \ldots x_{n}\right)$ consisting of the $n$ measured attributes of the waveform is associated to each object $y$ as considered in Table 2. Here the Sink node computes bitwise AND, and OR of the temporal and spatial pattern of readings as relayed. The superimposed and correlated information thus helps in reliable detection of target and with relay nodes, power management is achieved which ultimately results in enhanced network life time. Yu et al. $[13,18]$ have proposed a data reconstruction technique that was based on time series, historical data, spatial location and spatio-temporal correlation. According to $\mathrm{Yu}$ et al. $[13,18]$ data can be reconstructed by a method based on time-series, or on historical data, or on spatial location or on space correlation. Our approach of bitwise AND and OR operations performed on the data received by sink node helps in reconstructing data that fails to get reported to sink node due to a set of tampered nodes that we assume are introduced in the network. These tampered nodes may have been hijacked or could have been turned off voluntarily or may have simply died out over a span of time due to energy drainage. Reducing energy consumption is very crucial. Han et al. [19] have tried optimizing energy consumption in wireless sensor network by evaluating spatial and temporal coverage of sensor nodes. We use spatial and temporal coverage of nodes to detect any fault in sensors. Let us understand the following situation with the aid of set theory. Consider a network of wireless sensor nodes, $\mathrm{C}$ number of nodes report a data value (amplitude) at time $t, H$ number of nodes reported the data value at $t-1$, and $V$ number of nodes report the data value at $t+1$. Using temporal correlation the sink node can find the set of nodes that reported at both $t$ and $t \pm 1$. This can be represented in set theory as $\mathrm{n}(\mathrm{C} \cap \mathrm{H})$ where $\mathrm{n}(\mathrm{C} \cap \mathrm{H})$ stands for number of nodes in between $\mathrm{C}$ and $\mathrm{H}$ that report at time interval $\mathrm{t}$ and $\mathrm{t}-1$. Further the set of nodes that report at $t-1$ and $t+1$ would be $n(H \cap V)$, while the set of nodes that report at $t$ and at $t+1$ would be $n(C \cap V)$. Sink can employ bitwise AND operation to know the elements of the set $(C \cap H \cap V)$, being the set of nodes that report at, time $t-1, t, t+1$. Further it can also relate spatially by finding the set of nodes that belong to the set $(\mathrm{C} \cup \mathrm{H} \cup \mathrm{V})$ 
being the set of nodes that have reported to the sink at-least once in time $t-1, t$ and $t+1$ by using the following operation

$$
\mathrm{n}(\mathrm{C} \cup \mathrm{H} \cup \mathrm{V})=\mathrm{n}(\mathrm{C})+\mathrm{n}(\mathrm{H})+\mathrm{n}(\mathrm{V})-\mathrm{n}(\mathrm{C} \cap \mathrm{H})-\mathrm{n}(\mathrm{H} \cap \mathrm{V})-\mathrm{n}(\mathrm{C} \cap \mathrm{V})+\mathrm{n}(\mathrm{C} \cap \mathrm{H} \cap \mathrm{V})
$$

\section{Phase 2: relay nodes in bottleneck zone}

The topology under study has a central sink node. Such a topology leads to situation of load imbalance, where nodes near the sink node have to continuously forward packets and therefore drain their energy more quickly. If all the nodes just surrounding the sink node drain all their energy, the network will get disconnected. This situation needs to be avoided as far as possible.

Kaswn et al. [20] have suggested a mobile sink node that itself moves around the network and collects data from other nodes. However, this may not be a feasible solution for our problem. Wang et al. [21] have proposed a sleep schedule mechanism for all the nodes in the network. Their method prolongs the network lifetime but cannot be applicable to our problem because in our case nodes have to continuously sense data. We propose a solution to deal with this issue of load imbalance and energy drainage of nodes in bottleneck zone by scheduling the sleep and active cycles here. Let us assume R number of active and $\mathrm{R}^{\prime}$ number of sleep nodes in the bottleneck zone. Figure 3 illustrates these two sets $\mathrm{R}$ and $\mathrm{R}^{\prime}$ of relay nodes that forward packets to sink nodes in alternate clock cycle; such that $R \cap R^{\prime}=\{\phi\}$. Maintaining the schedule may be a slight overhead but it still improves the network lifetime.

\section{System model}

As per "Problem formulation" the topology contains n"n sensor nodes. But for simplicity and simulation point of view we have considered the model of 25 nodes, 5 cross 5 with the sink node in the center as shown in Fig. 4. The bottleneck zone is the immediate

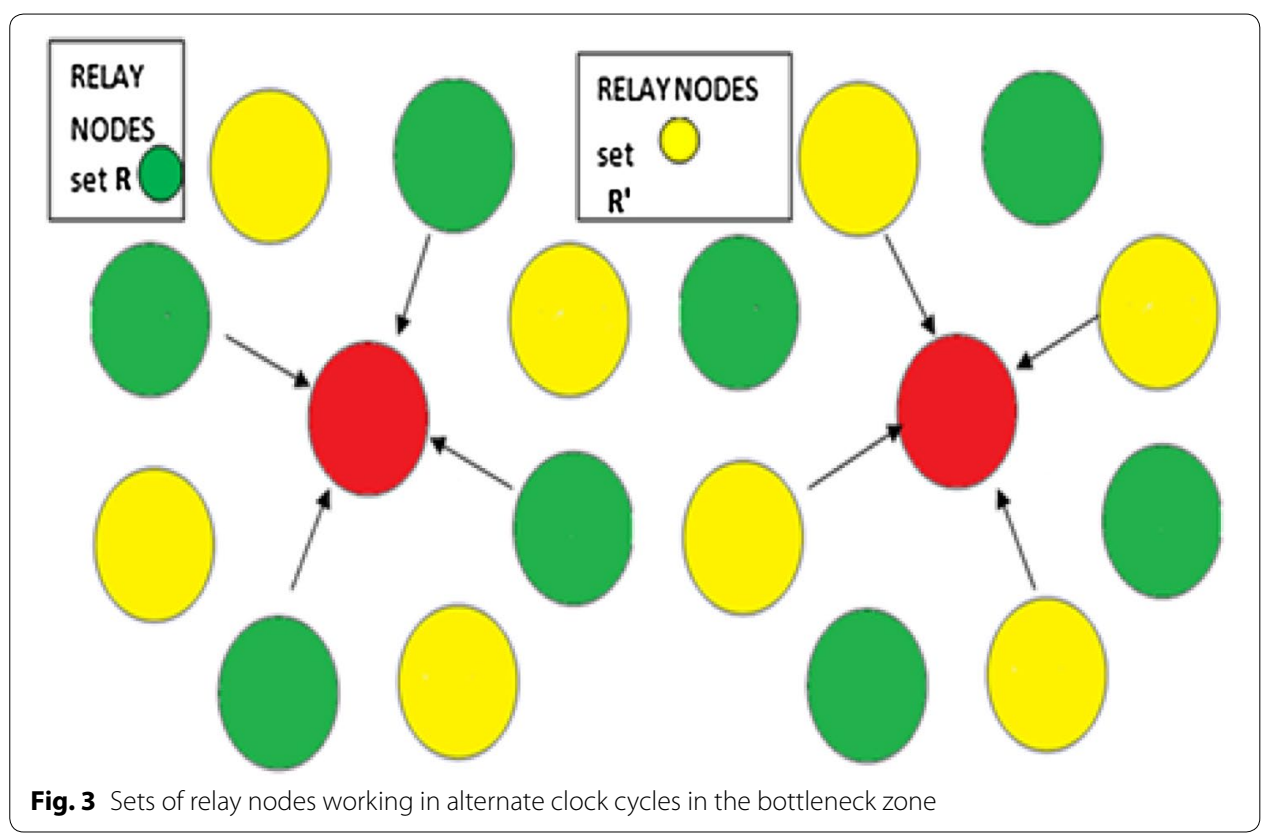


area around the sink node which remains active reporting the information to the sink node. The algorithm suggests two sets of relay nodes in the bottleneck zone that use data compression techniques and collate and forward the information that is received from its neighboring nodes. By allowing the first set of relay nodes to stay idle while the second set of relay nodes function, brings enhanced load sharing and there by increases the network lifetime. As shown in the figure below, the sink node's neighbors are all relay nodes that function in alternate clock cycles. From Fig. 4, it is seen that the dark node is sink node and all eight nodes surrounding the sink are relay nodes. The relay nodes work in alternate clock cycles to prolong the life time of the network. The sink node is on the ship while the remaining nodes are on the sea surface. However we have considered $10 \times 10$ nodes practically for simulation purpose in NS2 which is shown in Fig. 5 onwards.

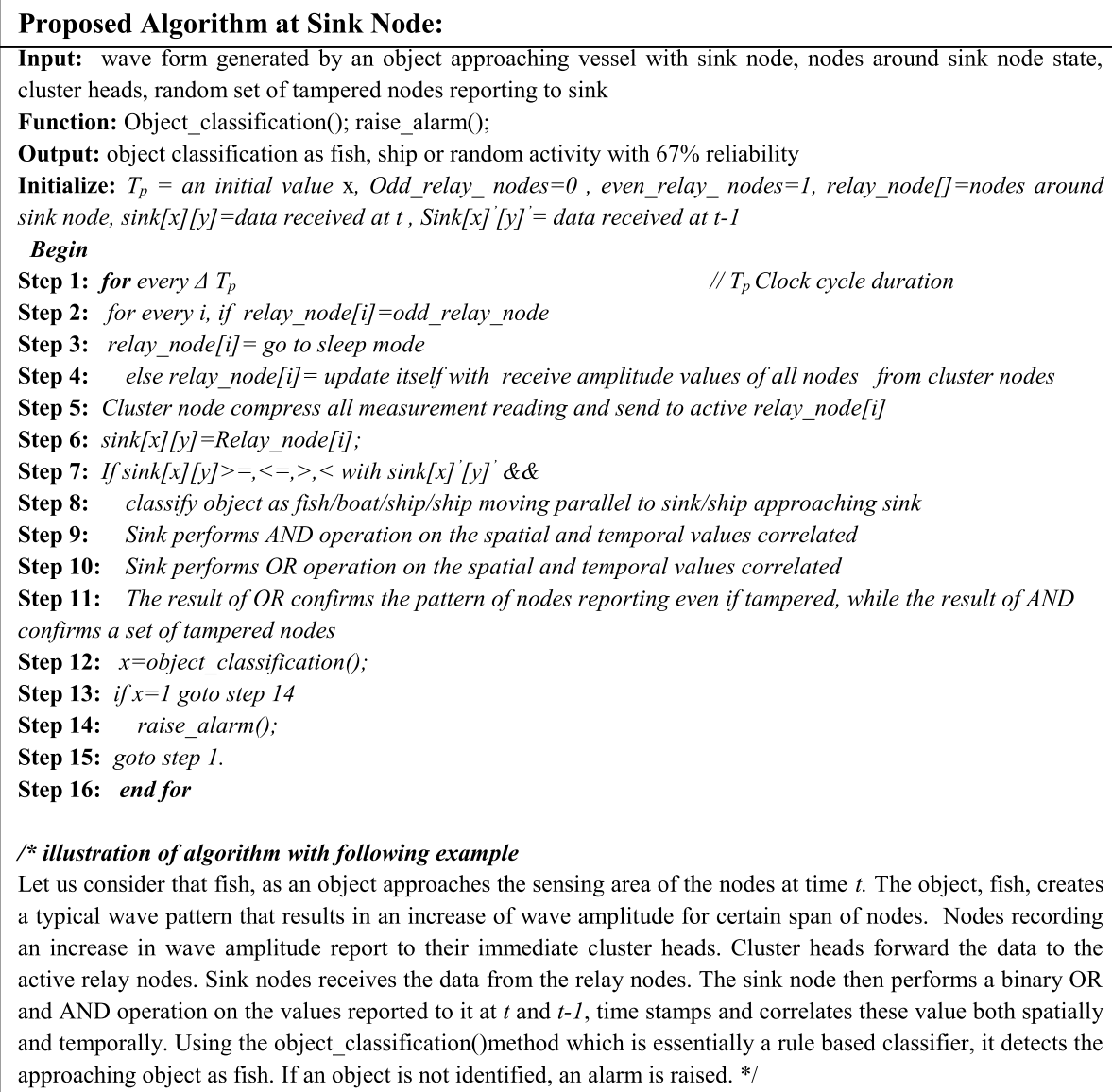

Each node of the setup is built with 3 axis accelerometer which detects the amplitude of the water level around it. These floating sensors further communicate with their cluster heads. Cluster level detection helps in learning the status of the nodes and of the network connectivity of clusters. Sink Node detects further by correlating the information received by the relay nodes. The information while being relayed could be tampered or changed. Information thereby gathered will fail to be reliable and faulty. We propose a technique that is $67 \%$ more reliable. 


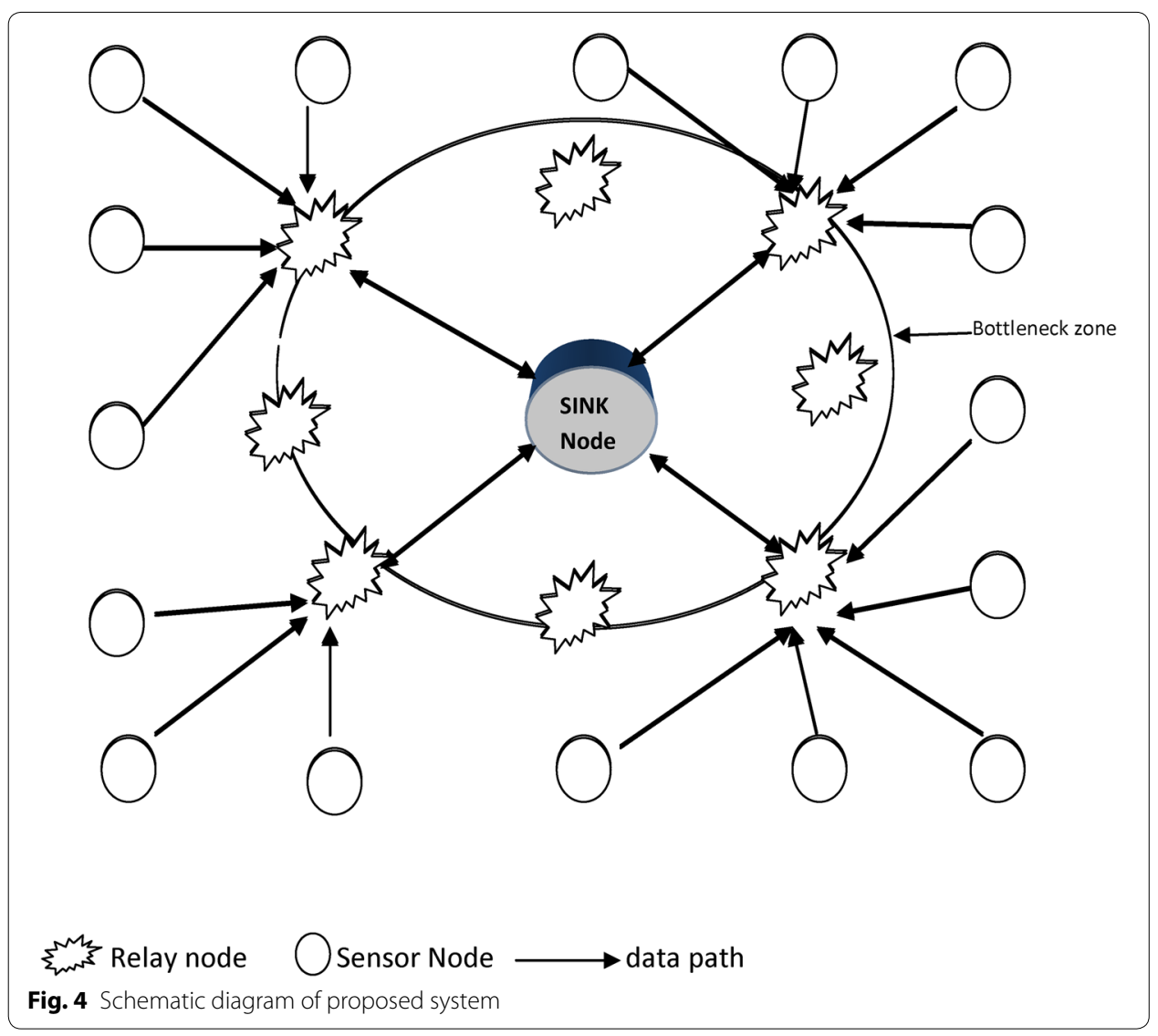

\section{Simulation results and analysis}

\section{Simulation set up}

Simulation has been carried out using NS2 with system configuration core to duo processor, $2 \mathrm{~GB}$ RAM and $2.66 \mathrm{GHz}$ frequency. The source data of test bed are wave patterns being generated in NS2 and subsequently are analyzed by the classification algorithm in Matlab. The results obtained are plotted across temporal and spatial values of nodes in three dimensional scales. Further the proposed object classification algorithm's performance parameters are studied in NS2.

\section{Classification of objects}

The pattern of wave trace generated by various objects helps in tracking and classifying the objects approaching or floating in surrounding. The temporal information is usually in the form of frame differencing, which highlights changing regions in consecutive frames. Given the object regions in the image, it is then required to perform object correspondence from one frame to the next so that the tracks could be generated. The aim of object tracking is to generate the trajectory of an object over time by locating its position in every frame of instant time. Object tracking also provides the complete region in the image that is occupied by the object at every time instant.

A grid of sensors is deployed around the ship which also contains the sink node. Figure 5 depicts the wave pattern generated by fish moving towards the sink. The $\mathrm{x}$ and $\mathrm{y}$ axis of the graph in Fig. $5 \mathrm{a}-\mathrm{c}$ indicate the nodes position in a two dimensional scale, 
while the $\mathrm{z}$ axis indicates the reading of wave amplitude being sensed by the node at a given node position $(x, y)$. It can be noted from Fig. $5 a-c$ that the fish is moving towards the sink. This can be known by the nodes reporting the increase in amplitude as a moving fish is likely to create. Under these conditions it has been assumed that the fish is moving with constant speed.

Figure 6 shows the span of nodes reporting the increase in amplitude to the sink node. With the proposed algorithm at the sink node, it can correlate the spatial and temporal values of the information received from the relay nodes. The span of nodes reporting increased in amplitude help in differentiating the object as boat, fish, etc.

The sensor readings are represented in a matrix for a given instance of time $t$ as given below, where the value represented is the height of amplitude being sensed by the senor in a given position along the $\mathrm{x}$ and $\mathrm{y}$ axis. For example a node located at position $(1,1)$ reports the sink that the height of wave amplitude is 22 units $(\mathrm{cm})$, node at $(1,2)$ reports 30 units where as node $(9,9)$ reports 20 units to the sink which is described in matrix

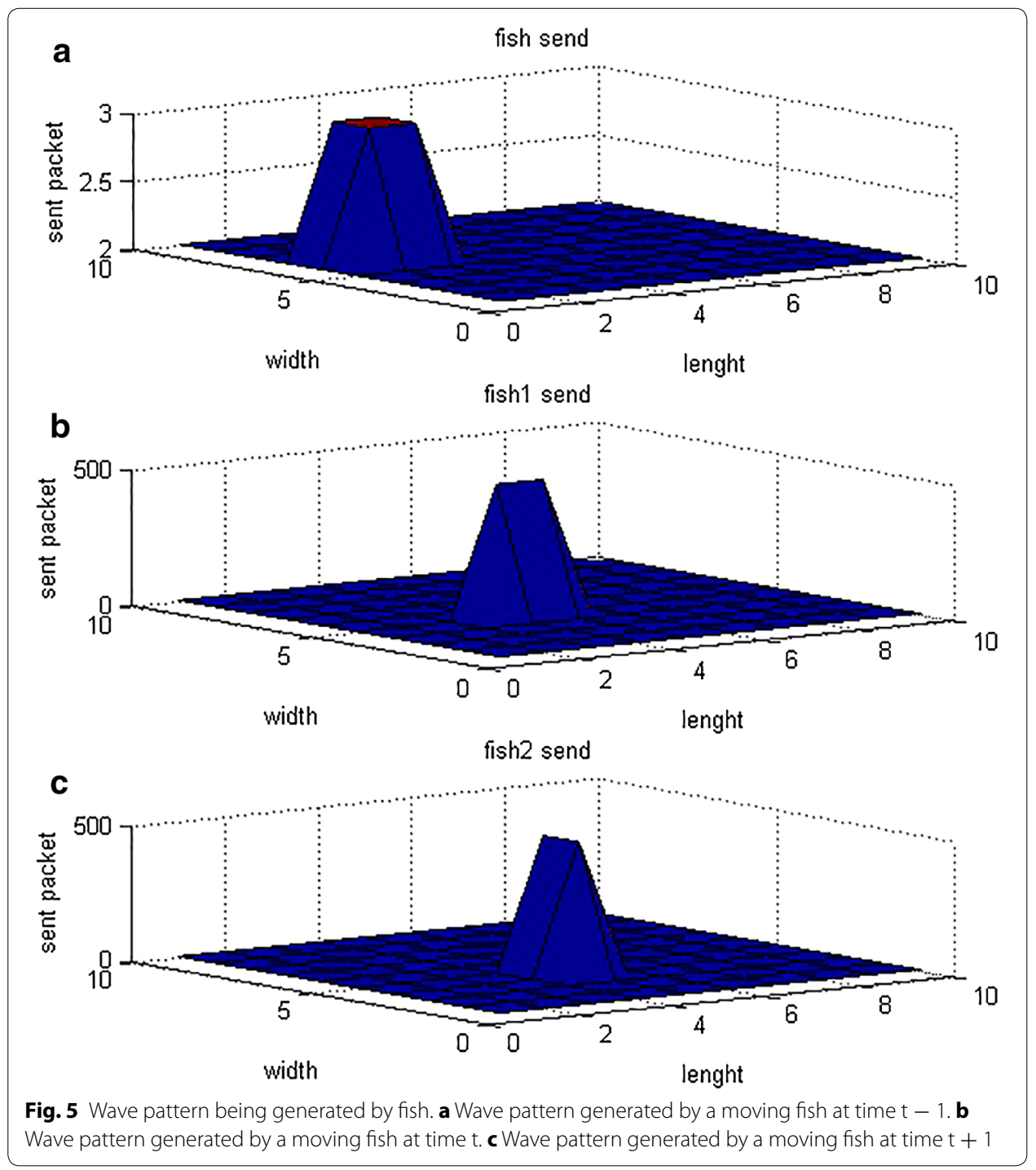




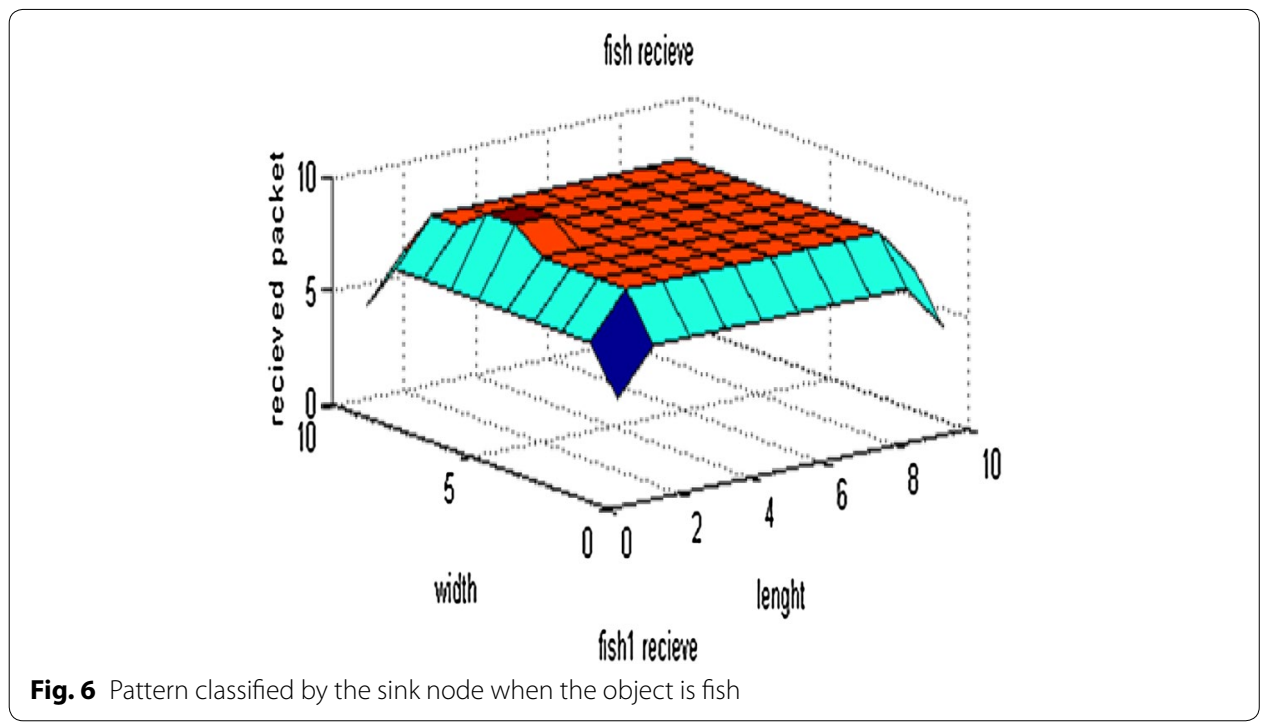

below for the Fig. 5a. These data sets are prototype for object, 'fish', in a normal day, but are vary in stormy or windy day.
22303020202020202020
30302220202020202020
30302120202020202020
20202020202020202020
20202020202020202020
20202020202020202020
20202020202020202020
20202020202020202020
20202020202020202020
20202020202020202020

In general the nodes report an amplitude of waves of height 20 units (can be considered in centimetres also) on the ocean surface. When an object as fish moves in the surrounding the nodes report the reading as depicted in above matrix. We use standard deviation to measure how close the set of data is to the mean value at time $t$. If data set has high standard deviation, then the values are spread out very much and that would be for an object of bigger dimension. If data sets have small standard deviation, the data points are very close to the mean and the object is likely to be small.

$$
\sigma=\sqrt{ } \sum\left(\mathrm{x}_{\mathrm{i}}-\bar{X}\right)^{2} / \mathbf{n}-\mathbf{1}
$$

where $\bar{X}$ is the mean. In the above example with the matrix mean are 20 . The mean is ideally calculated by the height of amplitude of waves on ocean surface. It may be higher on a stormy or windy day. We apply the Eq. 10 in the matrix values as generated by the moving object fish shown in Fig. 5a. The standard deviation row wise calculated is vector $\mathrm{S} 1$ as follows; S1: [4.1580, 4.1580, 4.1753, 0, 0, 0, 0, 0, 0, 0]. With this information it can be concluded that the region of the activity is restricted to the first 3 rows. The standard deviation for the moving object fish as shown in Fig. $5 \mathrm{~b}$ is calculated at time $t+1$ as well and is reported in vector S2; S2: [3.1552, 5.1208, 5.3759, 0, 0, 0, 0, 0, 0, 0]. Similarly Fig. 7a shows 


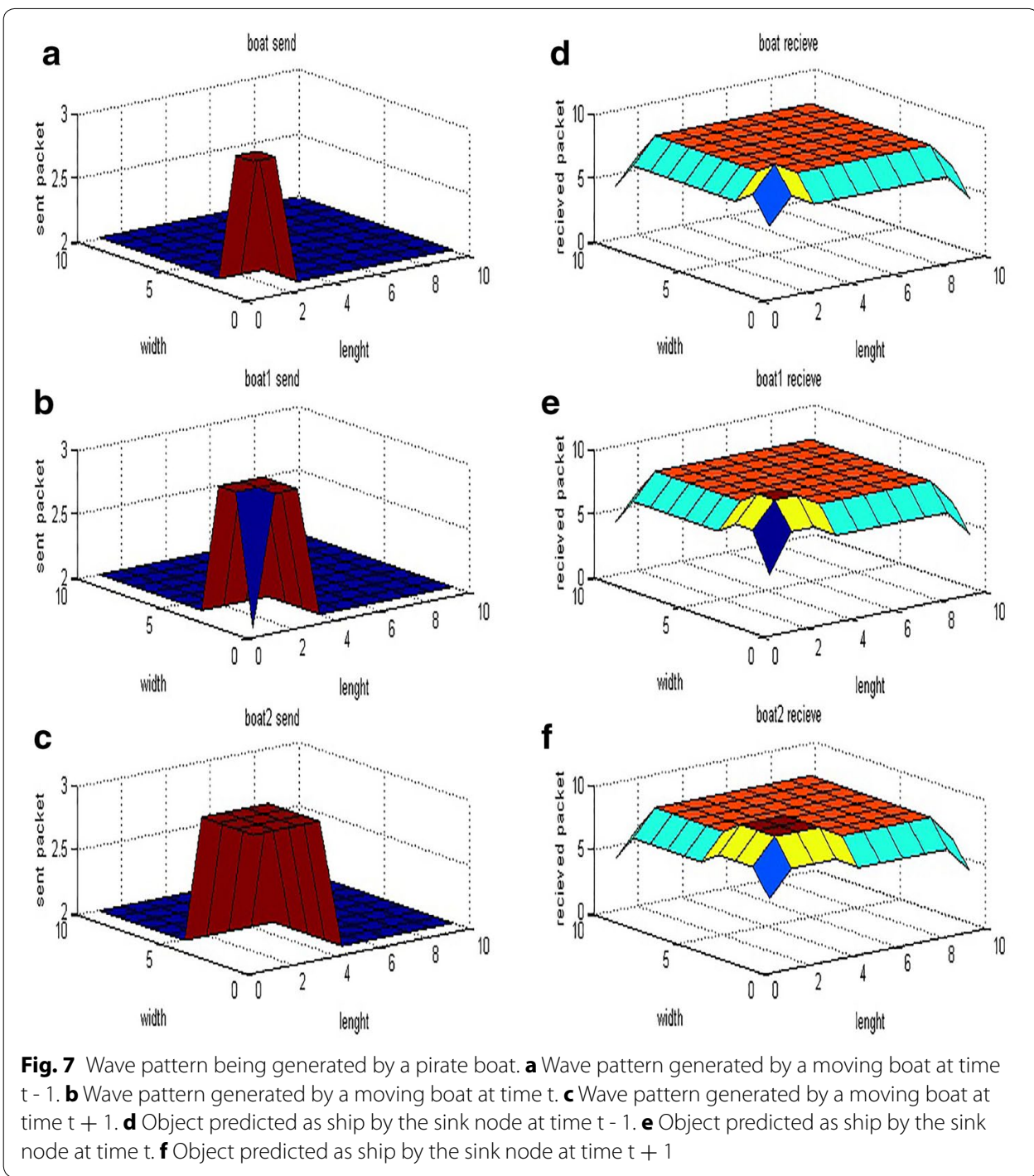

the waves generated by a pirate boat approaching the surrounding. Fig. $7 \mathrm{~b}, \mathrm{c}$ show the gradual movement of the pirate boat which is as per Eq. (1). Fig. $7 d-f$ depict the gradual information received at the sink node. The sink differentiate the object as an object much bigger than as reported in Fig. 5a-c. The Fig. $7 \mathrm{~d}-\mathrm{f}$ show three different colors which indicate the temporal values of the nodes. As a boat wave approaches the sink, it captures divergent and transverse waves as discussed and explained in Fig. 1. The pattern of waves generated by ships is a V-shaped pattern and is independent of the size and velocity of the ship. This V-shaped pattern is formed by two loci of cusps whose angle with the sailing line is $19^{\circ} 28^{\prime}$ in deep water and $54^{\circ} 44^{\prime}$ is the angle between the sailing line and the diverging wave crest line at the cusp of the local line as shown by Ursell [14]. Applying Eq. 10 on the data reading reported by nodes as a matrix at time $t$ as in Fig. 6a, b, the calculated standard deviation is described row wise in vector S3, S4 respectively; S3 $=[11.7379$, $12.5007,13.5827,15.1294,9.9359,0,0,0,0,0], \mathrm{S} 4=[10.5935,12.3581,13.8728,16.9247$, $13.9128,16.8655,0,0,0,0]$. Here standard deviation is much larger than matrix generated by Fig. $5 \mathrm{a}-\mathrm{c}$. It presumes that the object is much larger than a fish. 


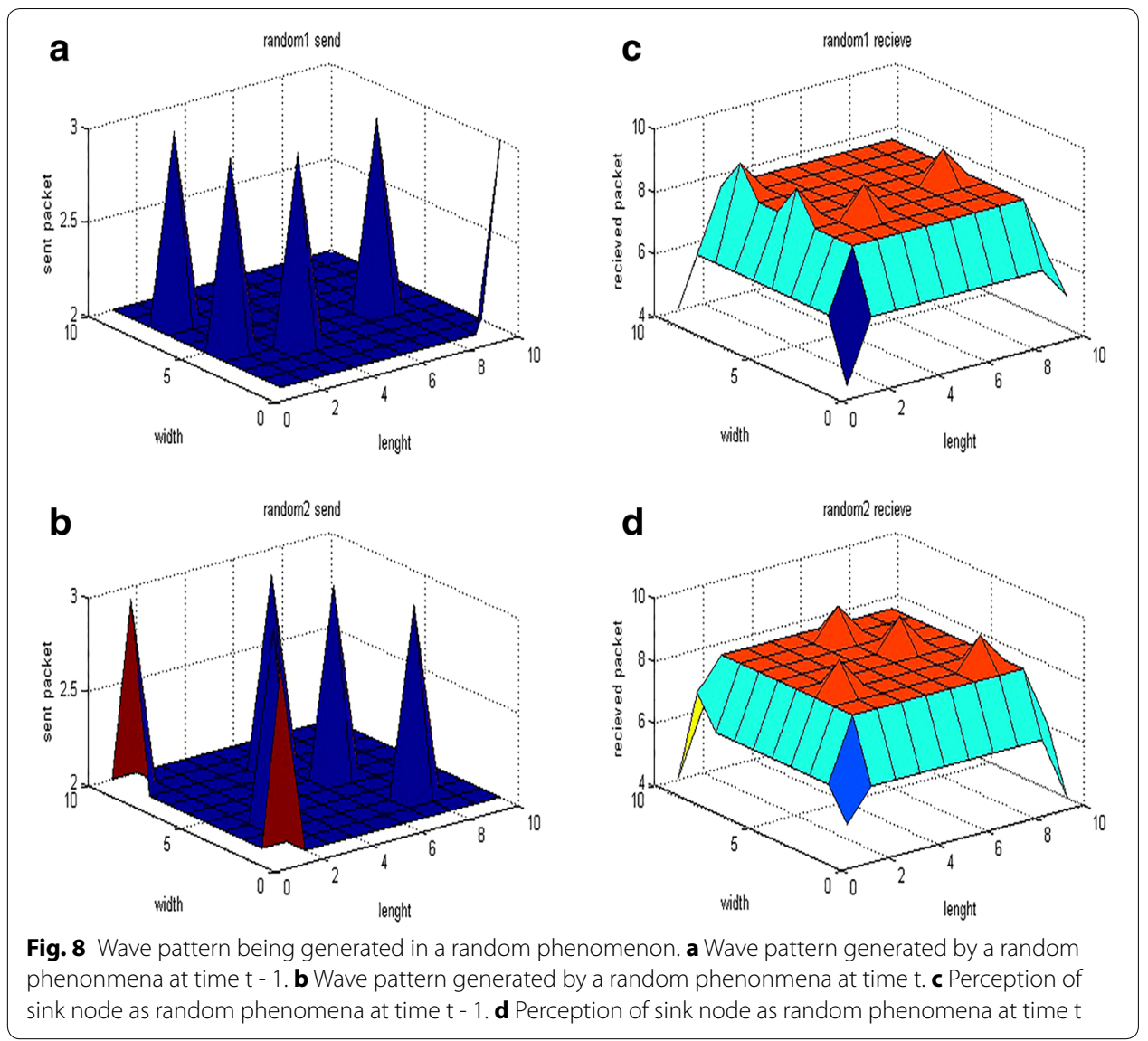

45454040202020202020

46464440202020202020

48494146202020202020

50505047202020202020

41414033202020202020

20202020202020202020

20202020202020202020

20202020202020202020

20202020202020202020

20202020202020202020

The sea surface is a bed for various types of organisms and other objects which create waves are considered as random phenomenon. These objects could approach the surrounding and would generate increase amplitude which is also reported to sink. Figure $8 \mathrm{a}, \mathrm{b}$ depict such random phenomenon. Figure $8 \mathrm{c}$, d depict the sink nodes perception of information as received from the relay nodes. We find the standard deviation using Eq. 10 for the matrix as obtained by Fig. 8a. The resultant vector S4: [0, 4.1110, 0, $6.3246,0,0,0,0,0,0]$. It standard deviation for the matrix as obtained from Fig. 8b: [0, $5.1110,0,6.725,0,0,0,0,0,0]$.

\section{The aspect of reliability}

We further study the reliability of the proposed object detection. It is likely that the nodes in the surrounding are tampered to report erroneous results further causing 
failures in detection and classification of objects. The proposed technique is more reliable as it computes bitwise AND and OR of the spatial and temporal values as received by the Sink node. It can predict the nodes that should have responded in due course of time but have not by reconstructing the inherent nature of waveform which is spatially and temporally correlated. Ran et al. [22] have used a similar approach where they fused traffic flow data received from multiple sensing locations to reconstruct the missing traffic data using full spatial and temporal information of traffic flow. We explore spatial temporal correlation for reliable object detection and reconstruction of its possible trajectory in progressing series of time. The proposed algorithm is validated for various objects and with various numbers of nodes reporting erroneous reading. We find that the object can be detected reliably $67 \%$ more than as reported by Luo et al. [7]. We assume that some of nodes are tampered and report erroneous values at an instance of time. However with the proposed technique super imposition of the spatial and temporal correlation of data, the success of detection percentage could be made more reliable. A wave that is generated by a moving abject will have smooth transitions. Data received at the sink node will thus have similar values that increase or decrease gradually from temporal aspect, while these changes will reflect spatially depending on the kind of wave generated. Attacks in wireless sensors network are node specific. Using spatial and temporal correlation we can disregard the effect of a malicious node. Spatial and temporal correlation is so inherent in many applications that it can be used to reduce the complexity of a system. Zhang et al. [23] have applied the temporal and spatial correlation of coding information of the previously coded frames for reducing the computational complexity of high efficiency video coding (HVEC) encoding.

Figure 9a depicts the increased amplitude of nodes in a two dimensional scale as reported in Fig. 5a. For better result and simulation point of view here 5 nodes are assumed to have been tampered and therefore report erroneous amplitude to the sink. Figure $9 \mathrm{~b}$ is the resultant graph of the superimposition of spatial and temporal values of nodes using Bitwise AND while Fig. 9c is the resultant graph of the superimposition of spatial and temporal values of nodes using Bitwise OR. It can be noted from the difference in Fig. 9b, $c$ that the nodes have been tampered and could raise an alarm. Ideally if the nodes have not been tampered the Fig. 9b, c would reflect the same readings. In Fig. 10a, varying number of tampered nodes resulting in an erroneous reading of amplitude sensed is reported to the sink node. The sink node employs the proposed algorithm, the result of bitwise AND is plotted in Fig. 10. Figure 10c is the result of bitwise OR of the super imposition of the spatial and temporal values of nodes.

The amplitude reported in Fig. 10a-c confirms that a wave of greater speed is reported which certainly reflects that the tracked object is not of a small object like fish. The span of the wave is confirmed in Fig. 10c while Fig. 10b confirms that some number of nodes have been tampered, this could also help in raising alarms. Figure 11a, b shows the simulation results when two boats approach each other with constant velocity are tracked in progressing instance of time. The $\mathrm{x}$ and $\mathrm{y}$ axis of graph indicate the nodes position on a two dimensional scale, the $\mathrm{z}$ axis indicates the amplitude sensed.

When two ships are moving parallel to each other with constant speed, the resultant waves are tracked as depicted in Fig. 12a, b in progressing instance of time. If two resultant waves being generated by boats meet in phase, then the resultant wave is amplified. 

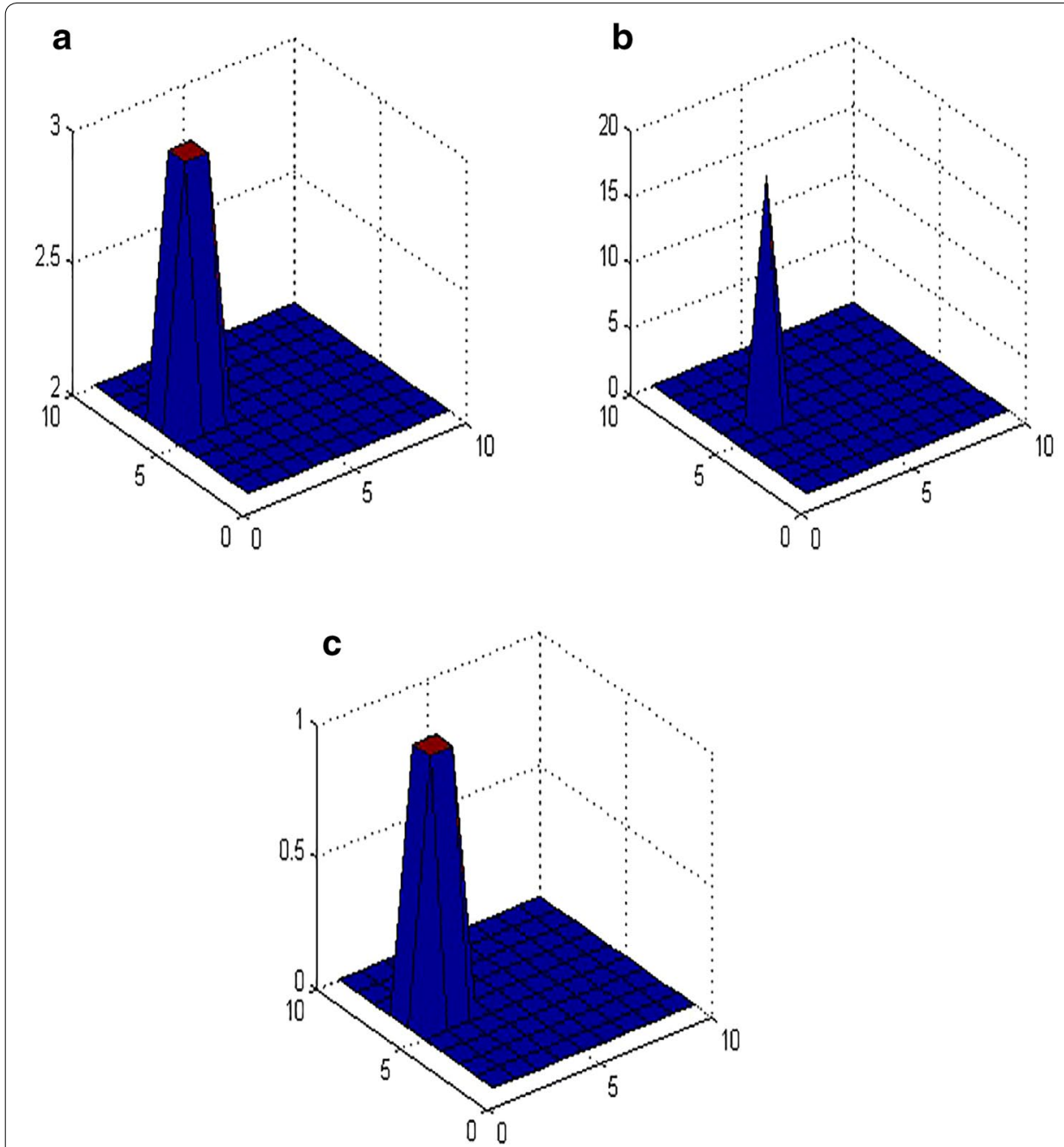

Fig. 9 Bitwise OR/AND of spatial and temporal values of nodes reporting the object as fish. a wave pattern generated by a moving fish at time t. b Bitwise AND on spatial and temporal values of (a). c Bitwise OR on spatial and temporal values of (a)

However if waves meet out of phase, resultant wave is nullified. This needs further research.

\section{Performance analysis}

In this paragraph the effectiveness of the proposed algorithm is compared with the only existing technique as proposed by Luo et al. The experiment is carried for various numbers of tampered nodes. Various scenarios containing objects like fish, boat and any random activity are simulated and results are examined. The performance analysis is based on target detection reliability and living nodes as time increases. However, jitter, throughput and delay are related to network lifetime. Figure 13 depicts the reliability of detection in percentage against the tampered nodes.

A tampered node can be defined as a node that is either turned off or is a node that sends wrong values so that object detection is inaccurate. We run the simulation by 

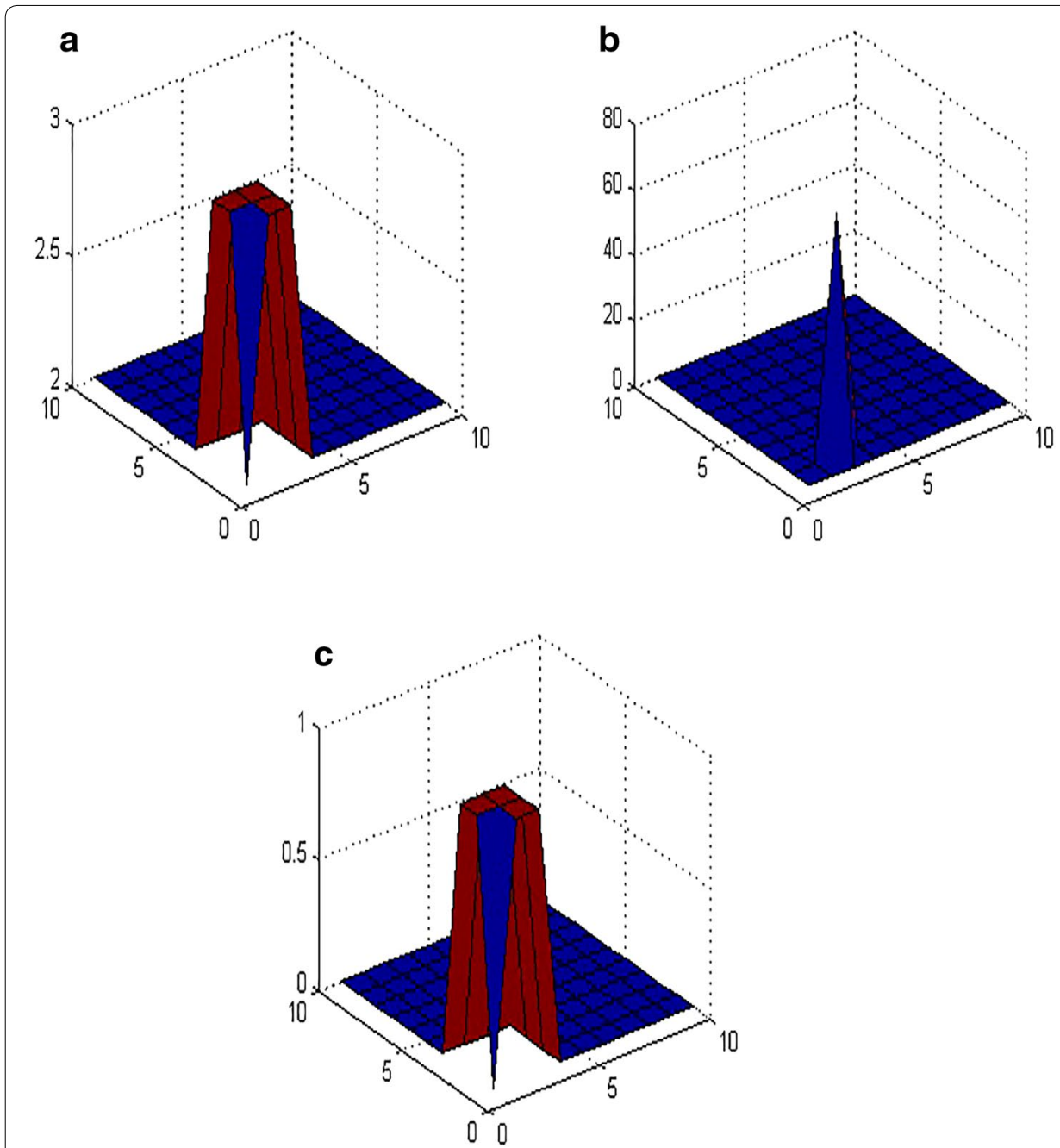

Fig. 10 Bitwise OR/AND of spatial and temporal values of nodes reporting the object as pirate boat. a Wave pattern generated by a moving boat at time t. b Bitwise AND on spatial and temporal values of (a). c Bitwise OR on spatial and temporal values of (a)

randomly affecting two nodes out of the 100 nodes considered in the simulation set up and record the success of the type of object detected. Later we successively increase the random number of affected nodes. From Fig. 13, it can be seen that both the proposed algorithm and the existing technique of Luo et al. [7], have high reliability initially, then the reliability is seen decreasing as number of tampered node increase. But reliability in our proposed algorithm is quite better than existing methodology of Luo et al. In other words it reflects that increase in tempered nodes reduces the reliability which is very natural. Simulation study suggests the average reliability of the proposed algorithm is $67 \%$ where as in case of existing algorithm it seems $42 \%$. The reason contributing to the better performance of our algorithm is due to the bitwise AND and OR of the spatial and temporal values of the node information. From Figs. 5c-e, 6c-e, 7c, d it can be noticed that the nodes just adjacent to the sink node are the nodes that require to perform constantly as they are the nodes situated in the bottleneck zone. The proposed 


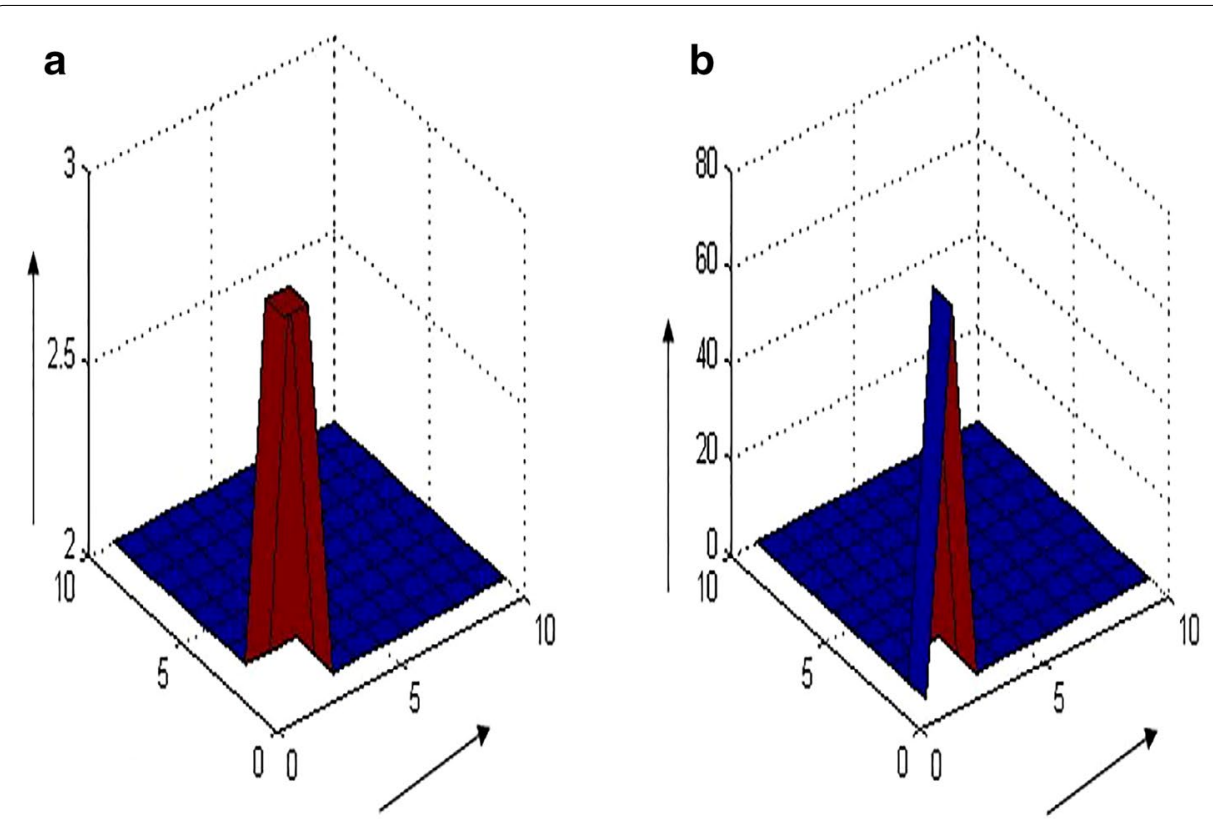

Fig. 11 Wave pattern for two pirate boat crossing each other. a Wave pattern generated by two pirates boats crossing each other at time $\mathrm{t}-1$. b Wave pattern generated by two pirates boats crossing each other at time $t$

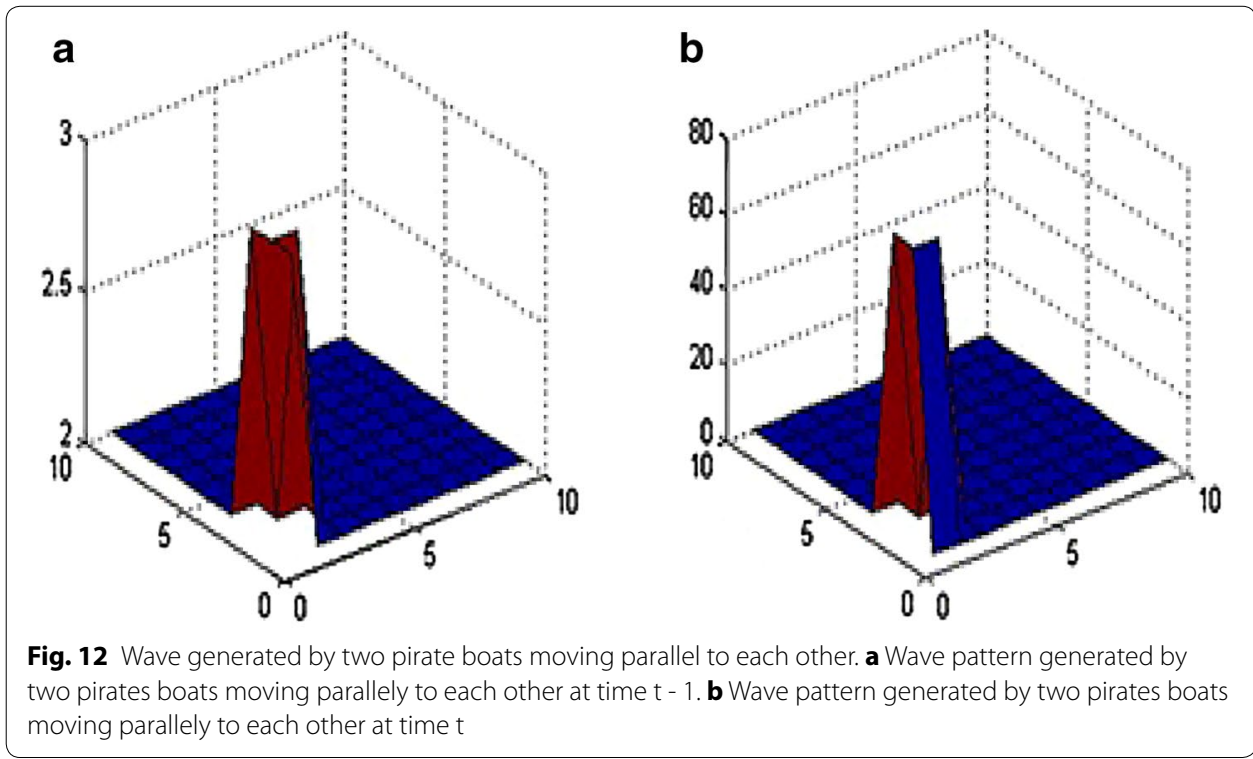

algorithm divides these nodes into two groups of relay nodes that function on alternate clock cycles, ensuring that network connectivity continues while network lifetime is also enhanced. The amount of living nodes over time is illustrated in Fig. 14. We analyze the power management and enhanced network life time that is achieved with the help of relay nodes in the bottleneck zone around the sink. By choosing to sleep on alternate cycles, the relay nodes save energy allowing the extension of their lifetime, and thereby contributing to the whole lifetime of the network. The existing approach consumes valuable node energy and shortens the network lifetime, however the proposed approach of 


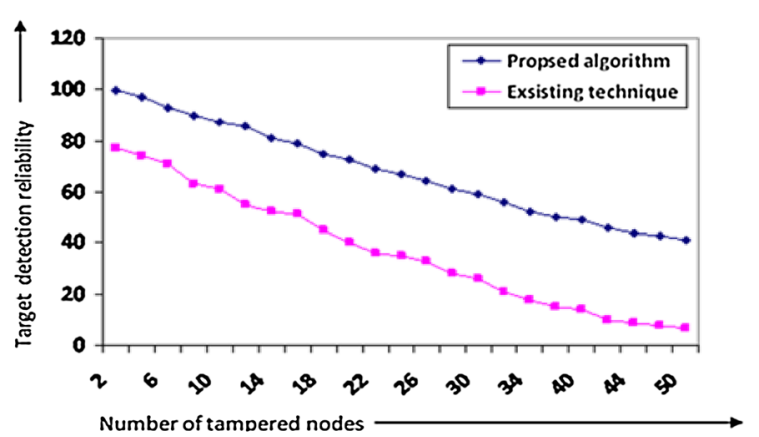

Fig. 13 Target detection reliability in \% across number of tampered nodes

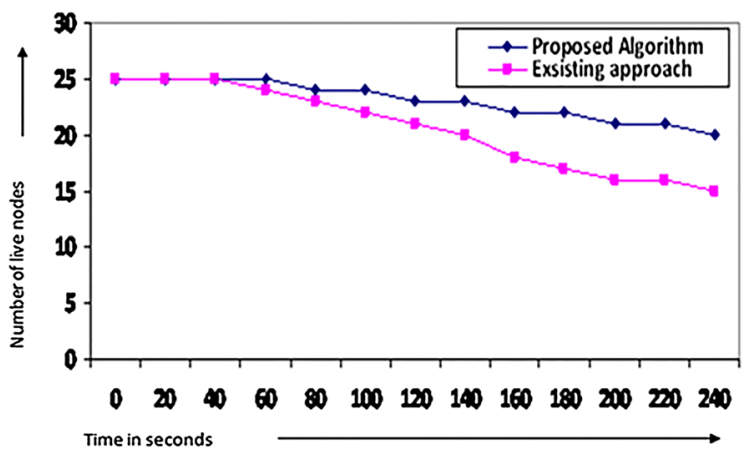

Fig. 14 Living nodes over time in seconds

alternate duty cycles for relay nodes brings elongated network lifetime, as nodes will live longer.

Figure 15 further displays the jitter, delay and throughput results of both existing technique that does not use relay nodes with our proposed technique of object classification based on waveforms and that employs relay nodes in the bottleneck zone along with bitwise AND and OR operations. To study these performance metrics, the simulation was carried out with various waveforms of different objects as inputs to the classification algorithm proposed here. Figure 15b illustrates the jitter of packets transmitted to the sink node in the existing technique, while Fig. 15a illustrates the jitter of packets with our proposed technique.

Jitter is defined as the varying delay of packets that arrive at the receiver. Jitter can be caused due to transmission errors, congestion and with varying number of hops to the reach the destination. Our approach yields lesser jitter with a maximum of $0.003 \mathrm{~ms}$ while with the existing technique, jitter reaches a maximum value of $0.009 \mathrm{~ms}$. The delay of the packets with our approach is plotted in Fig. 15e, f depicts the delay with existing technique. The yellow line in the delay Fig. 15e, f represents the delay of acknowledgement packets, while the green line indicates the delay of TCP packets. More than 1200 packets where generated in our approach, while with existing approach around 700 packets are generated. The delay TCP packets in our approach reduces gradually, where as in the case of existing approach the delay is almost same while the delay for 


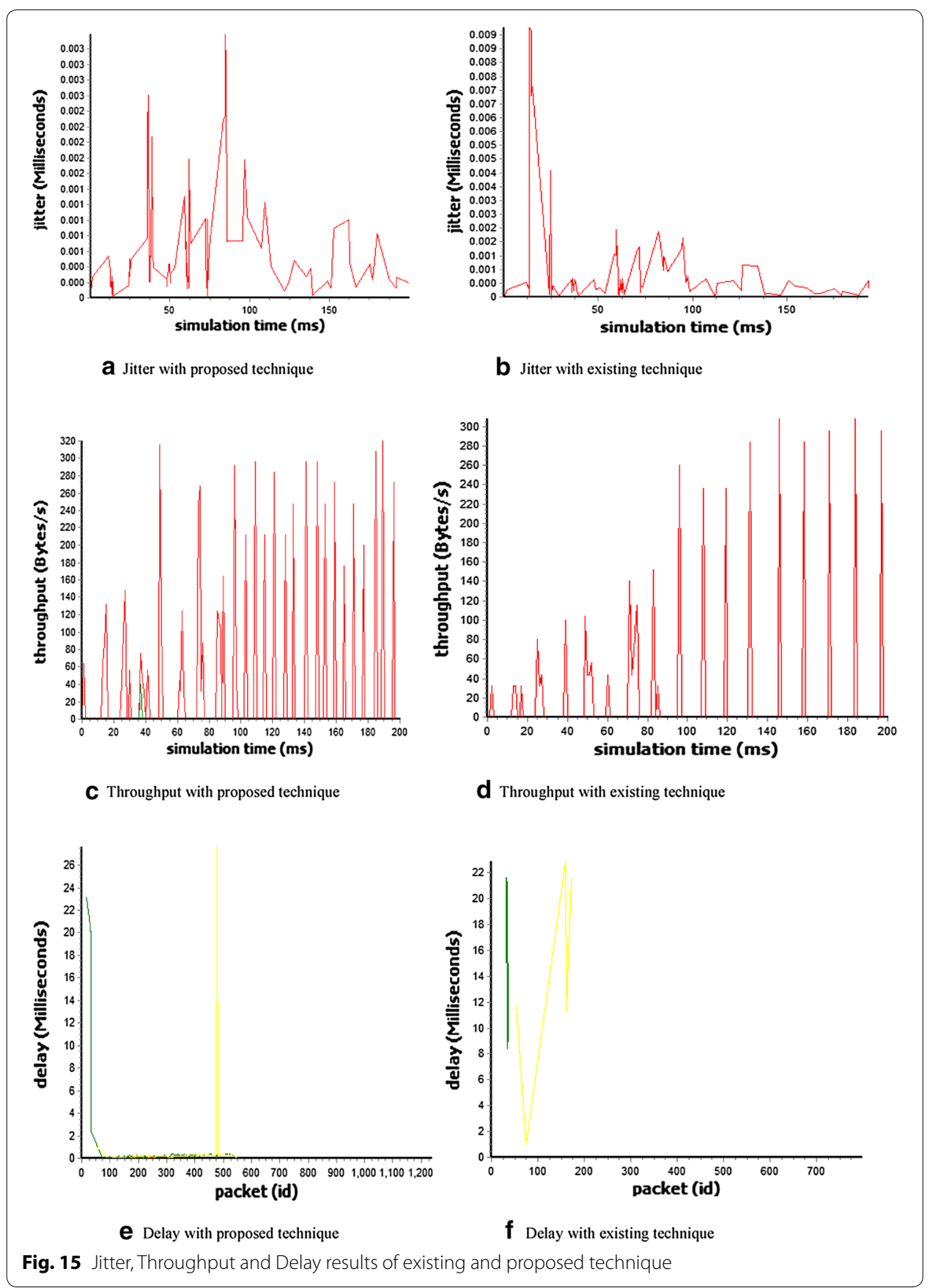

ACK packets is having no definite manner. It is quite unpredictable. The sudden delay of ACK packets in our approach occurs when there is a change in the duty cycle of the relay nodes. More number of packets is generated in our approach because of the need to coordinate the duty cycle of relay nodes and to ensure reliability. The Sink node performs bitwise AND and OR operation on the data received by sensor nodes both to find a wave pattern peculiar to each object using spatial and temporal correlation. We justify that though the overhead of packets is slightly higher in our approach, but we achieve a higher throughput and ensure the system as fault tolerant. Figure $15 \mathrm{c}$ depicts 
the throughput of both TCP and ACK packets in our approach while Fig. 15d depict the throughput of the existing technique. We achieve an average throughput of 24.78 bytes/s, where as 14.29 bytes/s is achieved by existing technique. We can justify from Fig. 15, which depicts the performance parameters of the proposed technique though it has a little overhead, but achieves better throughput and reduces jitter and delay. Our approach with duty cycled relay nodes avoids congestion in bottleneck zone and brings fair load distribution, thereby enhancing the overall network lifetime of the network.

\section{Conclusion}

Vessel monitoring systems which can carry an array of sensors with it for tracking objects approaching it by using spatial and temporal correlation of waves generated has been proposed earlier but was in very nascent stage and also failed to address the issues of load imbalance and fault tolerance. A lot more investigations will be needed for it to be commercial aspect. Here an attempt is taken forward in this direction. Our contribution is as follows: We develop a classification algorithm for identifying various objects approaching a moving ship. Objects like other ships moving in parallel and in opposite direction is also successfully detected with our approach. Our classifier is rule based and with proposed algorithm it identifies the objects correctly. We further identify the constraints of this mesh topology under study and propose a solution for reducing the energy drainage of the nodes in the bottleneck zone. We have employed bitwise operations on data values received at sink node for spatial and temporal correlation to make the system fault tolerant. The proposed technique helps in detecting data that has not been reported or has been compromised. Simulation results justify that our approach reduces jitter, and delay while also enhances the throughput and network lifetime, compared to the existing technique. The simulation has been carried out for a very small and regular topology; however it needs to be studied for different topologies. Also a study is needed on the minimum, maximum and an optimal number of nodes needed to achieve the said intrusion detection. An embedded design for such smart sensors is also left for the future work. Techniques that could help in knowing the velocity of the approaching object also need to be explored. Our system is incapable to learn and detect new objects and new patterns for generalization, it needs to upgrade the system further, is a challenging task.

\section{Abbreviations}

WSN: wireless sensor network; ORB: Oriented FAST and Rotated BRIEF; SAR: synthetic aperture radar; TCP: transport control packets; ACK: acknowledgement.

\section{Authors' contributions}

This section outlines the contributions of the authors towards the manuscript, Madhuri Rao who will be addressed as (MR) and Narendra Kumar Kamila as (NKK) further. MR had designed the proposed model and carried out simulations in NS2 simulation tool. MR further along with NKK carried out analysis and interpretation of trace file data in Matlab software. MR and NKK collectively drafted the initial manuscript and further revised it critically for projection of important intellectual findings. The authors have no political, personal, religious, ideological, academic, intellectual, commercial or any other non financial form of competing interests in relation to this manuscript. Both authors read and approved the final manuscript.

\section{Authors' information}

Narendra Kumar Kamila: He received his master degree from Indian Institute of Technology, Kharagpur and subsequently obtained his Ph. D. degree from Utkal University, Bhubaneswar in the year 2000. Prof. Kamila was also post doctoral fellow to University of Arkansas, USA. He has completed many projects sponsored by various sponsoring agencies. He has guided many M.Tech. and Ph.D. students. His research area includes wireless sensor networking, Adhoc-networking; Image processing, meta cognition, data privacy/security. 
Madhuri Rao: Has received her Bachelor Degree in Engineering from Biju Pattnaik University of Technology in the year 2005 and Master of Technology Degree in Computer Science from Bharath University, Chennai in the year 2008. She is currently pursuing her research work in wireless sensor network. Her research interest lies in the areas of Adhoc and Sensor Networking, parallel and distributed systems, mobile agent programming and mobile computing.

\section{Author details}

${ }^{1}$ Department of Computer Science \& Engineering, Institute of Technical Education \& Research, Siksha 'O' Anusandhan University, Bhubaneswar, Odisha, India. ${ }^{2}$ Department of Computer Science \& Engineering, C.V. Raman College of Engineering, Bhubaneswar, Odisha, India.

\section{Acknowledgements}

We would like to thank the editors and anonymous reviewers for their worthy comments and constructive criticisms for improvement of the manuscript.

The authors sincerely acknowledge the technical assistance of Mr. K. Vinod Kumar, Department of Computer Science \& Engineering, Siksha' $\mathrm{O}$ ' Anusandhan University during the implementation of the algorithm.

\section{Competing interests}

In the past 5 years the corresponding author has received salary from Siksha 'O' Anusandhan University.

As Siksha' $\mathrm{O}^{\prime}$ Anusandhan University is not financing the article processing fee and publication cost of this manu-

script, it may not in any way gain or lose financially from the publication of this manuscript, either now or in the future.

None of the authors hold any stocks or shares in any organization that may in any way gain or lose financially from

the publication of this manuscript, either now or in the future.

None of the authors hold or are currently applying for any patents relating to the content of the manuscript. The corresponding author receives salary from Siksha 'O' Anusandhan University but it does not hold and has neither applied for patents relating to the content of the manuscript.

\section{Availability of data and materials}

The Matlab and NS2 code of the simulation study can be made available if requested.

\section{Funding}

MR has been associated with Siksha 'O' Anusandhan University since last seven years and has been receiving salary as well. This research was carried out in the infrastructure support provided by Siksha 'O' Anusandhan University.

Received: 17 September 2016 Accepted: 21 March 2017

Published online: 15 May 2017

\section{References}

1. Rout RR, Ghosh SK (2013) Enhancement of lifetime using duty cycle network coding in wireless sensor network. IEEE Trans Wirel Commun 12(12):656-667. doi:10.1109/TWC.2012.111412.112124

2. Park T, Shi H (2015) Extending the lifetime of barrier coverage by adding sensors to a bottleneck region. 12th annual IEEE consumer communications and networking conference (CCNC), Las Vegas, pp 537-542. doi:10.1109/ CCNC.2015.7158031

3. Mohsin F (2011) Classification of Object Tracking Techniques in Wireless Sensor Networks. Wirel Sens Netw 3(4):121-124. doi:10.4236/wsn.2011.34014

4. Wu S, Yawen F, Zheng S, Yang H (2012) Object tracking based on ORB and temporal-spatial constraint. IEEE 5th international conference on advanced computational intelligence, Nanjing, pp 597-600. doi:10.1109/ICAl.2012.6463235

5. Kdouh H, Zaharia G, Brousseau C, Grunfelder G, Farhat H, Zein GE (2012) Wireless sensor network on board vessels. IEEE 19th international conference on telecommunications, Lebanon, pp 1-6. doi:10.1109/ICTEL.2012.6221242

6. Thattil V, Vasantha N (2011) Energy efficient approach to intruder detection in militarily sensitive border using wireless sensor networks. IEEE 3rd international conference on electronics computer technology, (ICECT), Kanyakumari, pp 42-46. doi:10.1109/ICETECH.2011.5941853

7. Luo H, Wu K, Guo L, Gu L, Lionel M Ni (2012) Ship detection with wireless sensor networks. IEEE Trans Parallel Distrib Syst 23(7):1336-1343. doi:10.1109/TPDS.2011.274

8. Li Y, Parker LE (2008) Intruder detection using a wireless sensor network with an intelligent mobile robot response. IEEE Southeast Con 08 Huntsville, pp 37-42. doi:10.1109/SECON.2008.4494250

9. Graziano DM, D'Errico M, Rufino G (2016) Ship heading and velocity analysis by wake detection in SAR images. Acta Astronaut 128(2016):72-82. doi:10.1016/j.actaastro.201607001

10. Zhai L, LiY, SuY (2016) A novel ship detection algorithm based on anomaly detection theory of SAR images, progress in electromagnetic research symposium (PIERS), Shanghai. doi:10.1109/PIERS.2016.7735144

11. Hastings JV (2012) Understanding maritime piracy syndicate operations. J Secur Stud 21(4):683-721. doi:10.1080/09 636412.2012 .734234

12. Rao M, Kamila NK, Kumar VK (2016) Underwater wireless sensor network for tracking ships approaching harbor. Proceedings of IEEE international conference on signal processing, communication, power \& embedded system, pp 467-471. ISBN: CFP16H12-PRT/978-1-5090-4619-5

13. YuT, Akhtar AM, Wang X, Shami A (2015) Temporal and spatial correlation based distributed fault detection in wireless sensor networks. Proceedings of the IEEE 28th Canadian conference on electrical and computer engineering, pp 1351-1355. doi:10.1109/CCECE.2015.7129475

14. Ursell F (2006) On Kelvin's ship-wave pattern. J Fluid Mech 8(3):418-431. doi:10.1017/S0022112060000700

15. Chwang AT, Chen Y (2003) Filed measurement of ship waves in Victoria Harbor. J Eng Mech 129(10):1138-1148 
16. Ogawa Y, Shimada N, Shirai Y, Kurumi Y, Komori M (2015) Temporal-spatial of knot-tying procedures using RGB-D sensor for training of surgical operation. 14th IEEE international conference on machine vision applications, Tokyo, pp. 263-266. doi:10.1109/MVA.2015.7153181

17. He J, Xu Z, Chen Y, Pan Z, Chih-Lin I (2014) Work in progress paper: network deployment and operation based on spatial and temporal traffic model. 9th international conference on communications and networking in China (CHINACOM), Maoming, pp 144-147. doi:10.1109/CHINACOM.2014.7054275

18. Yu F, Zhijie S (2015) Methods of real-time data screening and reconstruction for dynamic traffic abnormal data. 6th international conference on intelligent system design and engineering applications, pp 500-503. doi: 10.1109/ ISDEA.2015.130

19. Han C, Sun L, Xiao F (2016) An energy efficiency node scheduling model for spatial-temporal coverage optimization in 3D directional sensor network. IEEE Access 4:4408-4419. doi:10.1109/ACCESS.2016.2592184

20. Kaswn A, Nitesh K, Jana PK (2016) A routing load balanced trajectory design for mobile sink in wireless sensor networks. IEEE international conference on advances in computing, communications and informatics, pp 1669-1673. doi:10.1109/ICACCI.2016.7732287

21. Wang Y, Chen H, Wu X (2015) An energy- efficient SDN based sleep scheduling algorithm for WSNs. J Netw Comput Appl 59:39-45. doi:10.1016/j.jnca.2015.05.002

22. Ran B, Tan H, Wu Y, Jin JP (2016) Tensor based missing traffic data completion with spatial-temporal correlation. Stat Mech Appl 446:54-63

23. Zhang Q, Zhao J, Huang X, Gan Y (2015) A fast and efficient coding unit size decision algorithm based on temporal and spatial correlation. Opt Int J Light Electron Opt 126(126):2793-2798

\section{Submit your manuscript to a SpringerOpen ${ }^{\odot}$ journal and benefit from:}

- Convenient online submission

\section{Rigorous peer review}

- Immediate publication on acceptance

- Open access: articles freely available online

- High visibility within the field

- Retaining the copyright to your article

Submit your next manuscript at springeropen.com 